\title{
The Use of Original and Hybrid Flower Pollination Algorithm In Estimating The Parameters of Software Reliability Growth Models
}

\author{
Jamal Salahaldeen Alneamy $^{1} \quad$ Marwah Marwan Abdulazeez Dabdoob $^{2}$ \\ ${ }^{1,2}$ Software Engineering Department, Computer and Mathematics Science College, \\ University of Mosul, Iraq
}

Received Accepted

21/06/2018 15/08/2018

\begin{abstract}
In order to assess software reliability, many software reliability growth models (SRGMs) have been used for estimation of reliability growth. . In this work, the parameters of (SRGMs) were estimated by using Flower Pollination Algorithm (FPA). Then, the (FPA) was hybrid with Real Coded Genetic Algorithm (RGA) to obtain Hybrid FPA (HFPA).

The results that obtained from (FPA) are compared to the results of five algorithms: Particle Swarm Optimization (PSO), Artificial Bee Colony (ABC), the Dichotomous Artificial Bee Colony (DABC), Classic Genetic Algorithm (CGA) and the Modified Genetic Algorithm (MGA).

The results showed that (FPA) outperformed the rest of the algorithms in parameters estimating accuracy and performance using identical datasets. Sometimes, the (DABC) showed better performance than (FPA).

Other comparisons were made between (FPA) and (HFPA) and the results show that the hybrid algorithm outperformed the original one.
\end{abstract}

Keywords: Swarm Intelligence, Software Reliability Models. 


\title{
استعمال خوارزمية تلقيح الزهرة الاعتيادية والمهجنة في تخمين معلمات نماذج نمو وثوقية البرمجيات
}

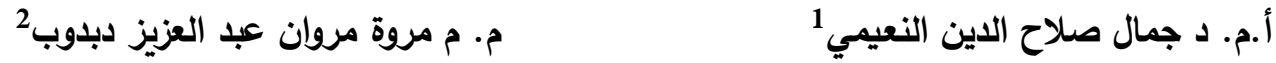 \\ 1,2 قسم هندسة البرمجيات/ كلية علوم الحاسوب والرياضيات/ جامعة الموصل \\ تاريخ الاستلام ت اريخ القبول \\ 2018/08/15 2018/06/21
}

\begin{abstract}
الخلاصة
Software ) من أجل تقييم وثوقية البرمجيات ، تم استعمال العديد من نماذج نمو وثوقية البرمجيات (Reliability Growth Models (SRGMs) باستعمال خوارزمية تلقيح الزهرة (FRGMs) (Real) Coded Genetic Algorithm(RGA)( للحصول على خوارزمية (FPA) الزهرة المهجنة (Hybrid FPA (HFPA). تمت مقارنة النتائج التي تم الحصول عليها من خوارزمية (FPA) مع نتائج خمس
\end{abstract}

خوارزميات وهي: خوارزمية سرب الطيور (Particle Swarm Optimization (PSO) ، خوارزمية مستعمرة

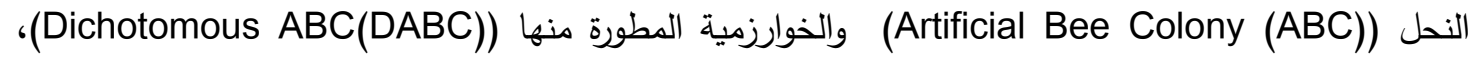
الخوارزمية الجينية الكلاسيكية (Classic Genetic Algorithm (CGA) والخوارزمية المحدثة منها .(Modified Genetic Algorithm (MGA)) أظهرت النتائج تفوق خوارزمية (FPA) على باقي الخوارزميات في تخمين المعلمات من إذ الدقة والأداء وباستعمال بيانات منطابقة. في بعض الأحيان ، أظهرت خوارزمية الـ (DABC) أداء أفضل من (FPA). وبالتالي تم إجراء مقارنات أخرى بين (FPA) و (HFPA) وأظهرت النتائج تفوق الخوارزمية المهجنة على الخوارزمية الأصلية من إذ الدقة وسرعة الأداء. 


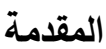

تعرف وثوقية البرمجيات بـ "كيفية تحقيق البرنامج للمتطلبات المرجوة " وتعرف أيضاً "إحتمالية عمل

البرنامج بدون فشل لمدة معينة من الوقت وفي بيئة محددة" [1].

إن محاولة معرفة هل أن البرنامج الذي سيسلم للزبون موثوق أم لا من الأمور الصعبة, فمن الممكن

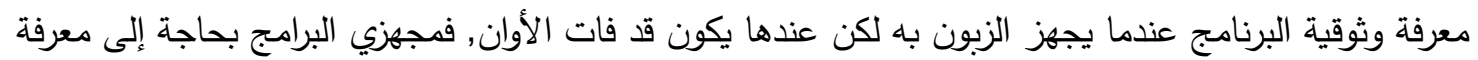

وثوقية البرنامج قبل تجهيز الزبون به. إن نماذج وثوقية البرمجيات تزودنا بمثل هذه المعلومات قبل التسليم [2].

خلال العقود الاربعة الاخيرة, تم اقتراح وتحليل العديد من نماذج نمو وثوقية البرمجيات لقياس نمو

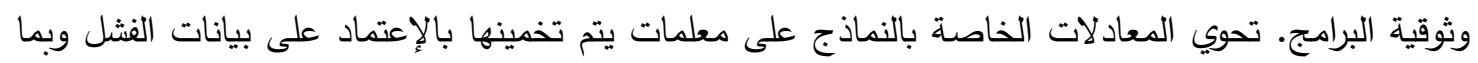

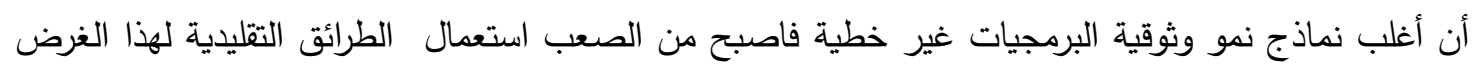

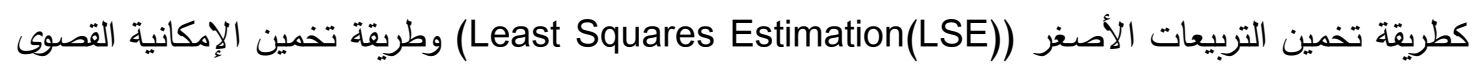
(3) (Maximum Likelihood Estimation (MLE)) طرائق اخرى لتخمين الوثوقية للمعادلات غير الخطية فكان التوجه لخوارزميات ما وراء الحس هن (تتضمن: الخوارزميات التطورية, خوارزميات اساسها الظواهر الفيزيائية وخوارزميات ذكاء السرب) والتي لاقت شعبية كبيرة في هذا المجال[4].

في هذا العمل ، سنستعمل خوارزمية من خوارزميات ذكاء السرب وهي خوارزمية تلقيح الزهرة (FPA) لتقدير معلمات الـ (SRGMs), ثم تهجين الـ (FPA) مع الخوارزمية الجينية المشفرة بقيم حقيقية (RGA) لتحسين أداء التخمين.

\section{1. نماذج نمو وثوقية البرمجيات}

في منتصف السبعينيات ،حققت الدراسات في نماذج نمو وثوقية البرمجيات تقدمًا كبيرا ، وقد تم بالفعل استعمال العديد من نماذج الوثوقية في هذا المجال[3]. ان الهدف من الـ (SRGMs) هو التنبؤ بسلوك الفثل المستقبلي ، والتي تستعمل الوقت بين حالات الفشل أو عدد الفشل الملاحظ في فترة زمنية محددة كبيانات لها [5] لهول.

(Non- هنالك العديد من نماذج نمو وثوقية البرمجيات لكن تعد نماذج عملية بواسون غير المتجانسة Homogeneous Poisson Process (NHPP))

الخطية [6].

هذه النماذج تساعد مهندسي البرنامج لإتخاذ القرار ما إذا وصلت وثوقية البرنامج إلى حد مقبول وتحديد

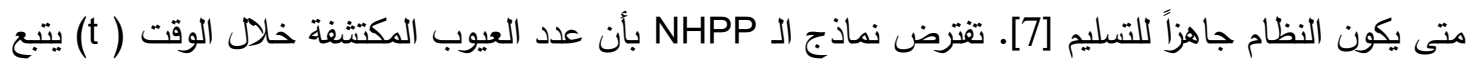
بدالة قيمة المتوسط (t) ب(t) ويؤدي إثتقاق دالة قيمة المتوسط إلى (t) والتي هي كثافة الفشل للبرنامج

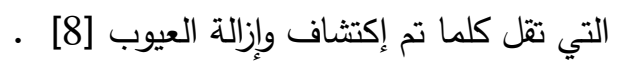


هنالك العديد من نماذج الـ (NHPP) استعملنا اربعة منها وهي نفسها المستعملة في الدراسات السابقة التي

$$
\text { سيتم المقارنة معها وكما موضح في الجدول ادناه: }
$$

الجدول (1) : نماذج الـ SRGM المستعملة في البحث.

\begin{tabular}{|c|c|c|}
\hline النموذج & دالة قيمة المتوسط & المخمنة \\
\hline G-Om & 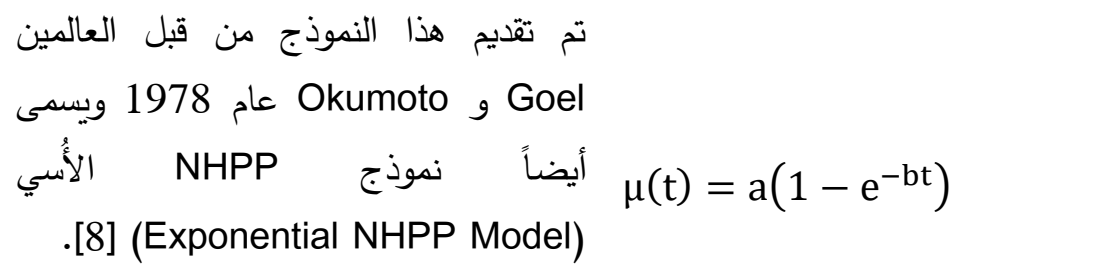 & $a, b$ \\
\hline POWm & 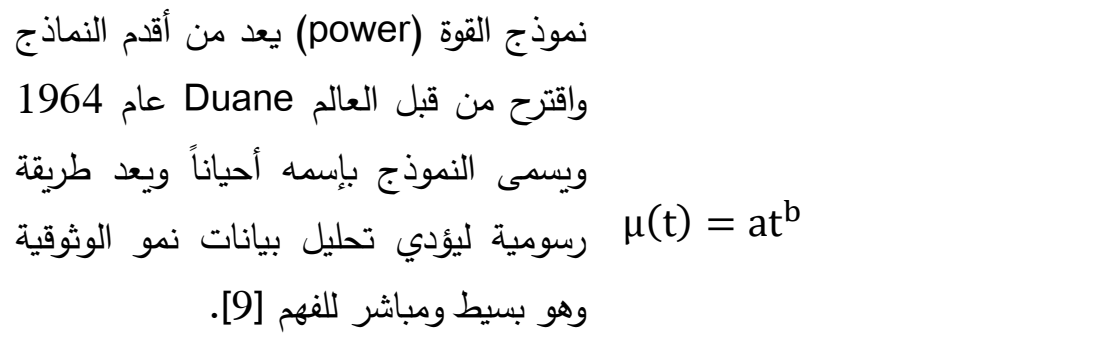 & $a, b$ \\
\hline DSSm & 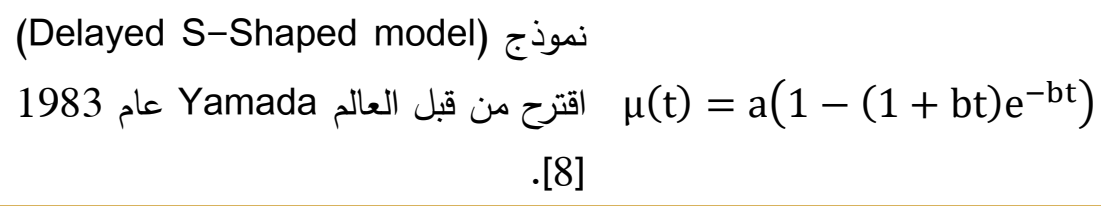 & $a, b$ \\
\hline INFSm & 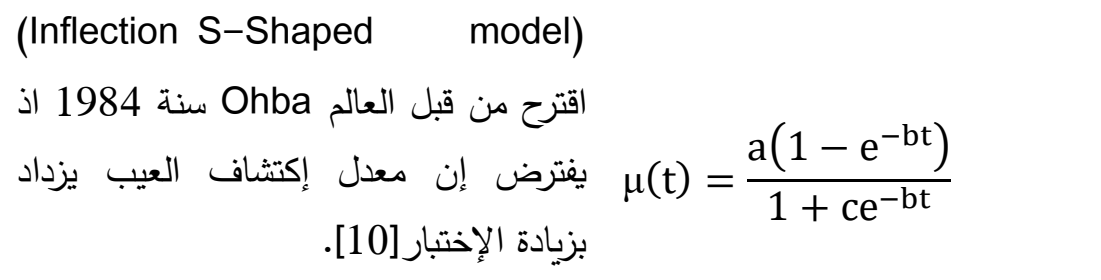 & $\begin{array}{l}a, b \\
\text { and } c\end{array}$ \\
\hline
\end{tabular}

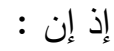

a > 0

b > 0

t : تمثل وقت حصول الفشل.

$\mathrm{c}=\frac{1-\mathrm{r}}{\mathrm{r}}$ 
اذ ان r> > > 1 > : تمثل معدل الإلتواء . 


\section{الجانب العملي}

سنتطرق في هذا الجانب الى الخوارزميات التي تمت المقارنة معها اضافة الى الخوارزميات المستعملة في هذا العمل وتفاصيل كل منهم.

1- الخوارزميات التي تمت المقارنة معها:

1-1 الخوارزميات ABC,PSO و AABC

تم استعمال خوارزمية (PSO) من قبل الباحث Sheta [11] اما خوارزميتي ABC و ABCD فقد DABC استعملت من قبل Ajith Abraham وآخرون [12] لتخمين معلمات نماذج وثوقية البرمجيات.

1-1-1-1 البيانات المستعملة:

تم استعمال مجموعة البيانات التي تم الحصول عليها من قياس فثل النظام أثناء الاختبار في تطبيق

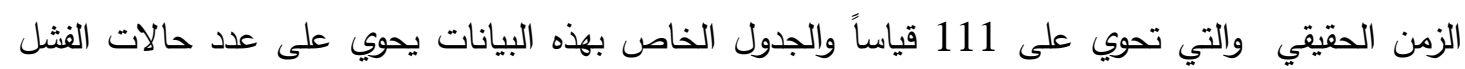

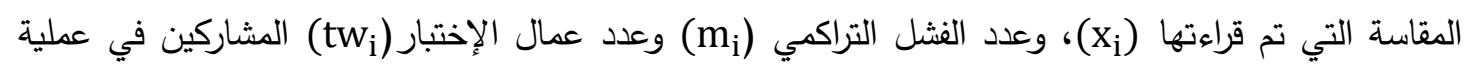
الإختبار ، ولقد تم قياس مجموعة البيانات هذه بالأيام والتي عددها (111) يوم. تم استعمال للتثريب و 30\% من البيانات للإختبار وسيتم تسيمة هذه البيانات بالبيانات الأولى.

1-1-2-1 دالة اللياقة:

تم استعمال دالة الجذر متوسط مربع الخطأ (RMSE) لإعطاء قيمة اللياقة لكل حل وتم استعمالها

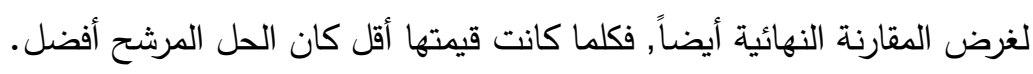

$\operatorname{RMSE}=\sqrt{\frac{1}{\mathrm{~N}} \sum_{\mathrm{i}=1}^{\mathrm{N}}\left(\mathrm{m}_{\mathrm{i}}-\mu_{\mathrm{i}}\right)^{2}}$

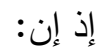

N : تمثل عدد القياسات المستعملة لتخمين معلمات النموذج. mi : تمثل عدد الفشل الملاحظ التراكمي.

年

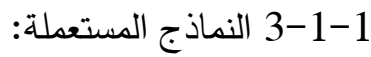

تم استعمال ثلاثة نماذج وهي:(GOW), (PSS), و(DSS).

1-2 خوارزميات

Chao-Jung Hsu من قبل الباحثين

و Chin-Yu Huang [13] ومقارنتها بالخوارزمية الجينية التقليدية (CGA) التي إستعملها الباحثان .Tohma ginohara 


$$
\text { تم استعمال ثلاثة أنواع من البيانات (Data set) وكما مبين في الجدول أدناه. }
$$

\begin{tabular}{|c|c|c|c|}
\hline وصف البيانات & عدد حالات الفشل & عدد أسابيع الإختبار & مجموعة البيانات \\
\hline $\begin{array}{c}\text { تطبيقات اللغات البرمجية ( البيات البمعة من اختبار } \\
\text { (database application }\end{array}$ & 328 & 19 إسبوع & DS1 \\
\hline برمجي من اربع اصدرات الاصدار الاول لنظام & 100 & 20 إسبوع & DS2 \\
\hline بيانات اختبار الشبكة اللاسلكية & 181 & 34 إسبوع & DS3 \\
\hline
\end{tabular}

إذ يحوي كل جدول على إسبوع الإختبار وعدد الفشل التراكمي (mil). وسيتم تسمية هذه البيانات بمجموعة البيانات الثانية. 1-2-2 دالة اللياقة:

Least Squares ) تم استعمال دالة لياقة مستتدة على طريقة تخمين التربيعات الأصغر

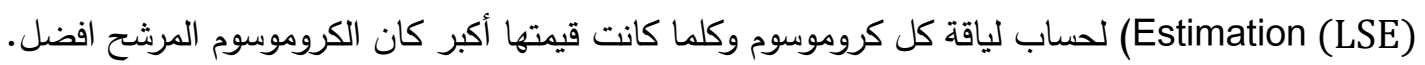
fitness $_{\mathrm{LSE}}=\frac{1}{\sum_{\mathrm{i}=1}^{\mathrm{N}}\left(\mathrm{m}_{\mathrm{i}}-\mu_{\mathrm{i}}\right)^{2}}$

وتم استعمال متوسط مربع الخطأ (Mean Square Error(MSE) لغرض المقارنة النهائية والتي كلما كانت أقل كانت أفضل. $\operatorname{MSE}=\frac{1}{\mathrm{~N}} \sum_{\mathrm{i}=1}^{\mathrm{N}}\left(\mathrm{m}_{\mathrm{i}}-\mu_{\mathrm{i}}\right)^{2}$

N : تمثل عدد القياسات المستعملة لتخمين معلمات النموذج. : تمثل عدد الفشل الملاحظ التراكمي. تمثي. بi

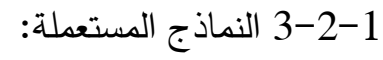
تم استعمال نموذجين(EXP) و (INFS). 2- الخوارزميات المستعملة في البحث: 
Flower Pollination Algorithm(FPA)

2-2 خوارزمية تلقيح الزهرة

تعد خوارزمية تلقيح الزهرة إحدى الخوارزميات المستوحاة من الطبيعة والتي إستُلهِمت من عملية تلقيح

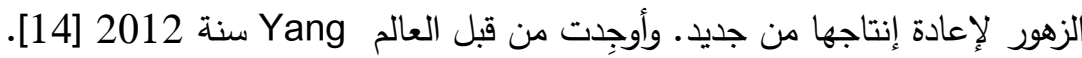

يمكن أن يأخذ التلقيح شكلين رئيسيين: حيوي (Biotic) وغير حيوي(Abiotic), حوالي 90\% من

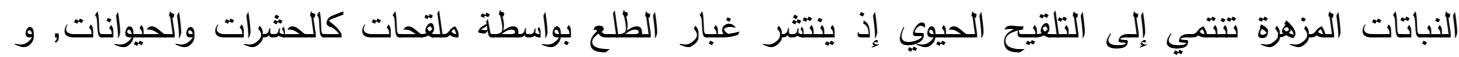
حوالي 10\% من التلقيح يكون غير حيوي أي لا يحتاج ملقحات بل تقوم الرياح والمياه بالمساعدة في التلقيح.

يتم التلقيح بطريقتين: التلقيح الذاتي (Self-Pollination): يحصل عندما حبوب لقاح من زهرة تلقح الزهرة نفسها أو زهرة أُخرى على النبات نفسه. التلقيح الخلطي (Cross-Pollination): يحصل عندما تتتقل حبوب لقاح من زهرة على نبات إلى زهرة أُخرى من نبات آخر [15].

\section{Rules Of FPA}

FPA قواعد خوارزمية 1-1-2

يمكن أن تُوصَف ثبوتية الزهرة وسلوك الملقحات في عملية التلقيح بالقواعد الأربع الآتية [14]:

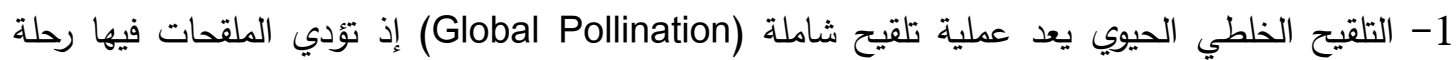
2- التلقيح الذاتي غير الحيوي يعد تلقيحا محليا (Local Pollination).

3- يمكن إعتبار ثبوتية الزهرة بأنها إحتمالية إعادة الإنتاج والتي تتتاسب حسب تثابه إثثين من الزهور المعنية. 4- التبديل أو التفاعل بين التلقيح الثامل والتلقيح المحلي يمكن أن يُسيطَر عليه من خلا إلد إحتمالية التبديل $\cdot p \in[0,1]$

\section{2-2-2 التمثيل الرياضي للتلقيح الثامل والتلقيح المحلي}

\section{Mathematical Representation Of Global And Local Pollination}

يعد التلقيح الثامل والتلقيح المحلي الخطوات الرئيسة لخوارزمية تلقيح الزهرة ـ ففي التلقيح الثامل تحمل حبوب اللقاح بواسطة الملقحات كالحشرات, وحبوب اللقاح يمكن أن تسافر لمسافات بعيدة لأن الحشرات يمكنها على الأغلب الطيران والتحرك لمدى طويل, لهذا تمثل القاعدة رقم 1 والقاعدة رقم 3 رياضياً كالآتي :[14] $x_{i}^{t+1}=x_{i}^{t}+\gamma L(\lambda)\left(x_{i}^{t}-x_{\text {best }}\right)$ 
أما في التلقيح المحلي فيكون التلقيح ذاتياً أي بين زهور على النبات نفسه, إذ تمثل القاعدة رقم 2 والقاعدة رقم 3 رياضياً كالآتي: - الشي:

$x_{i}^{t+1}=x_{i}^{t}+\epsilon\left(x_{j}^{t}-x_{k}^{t}\right)$

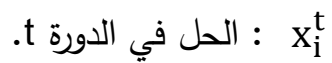

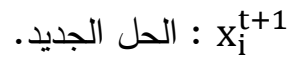

جبوب لقاح من زهور مختلفة على النبات نفسه إذ k, : مؤشرات مختارة عشوائياً.

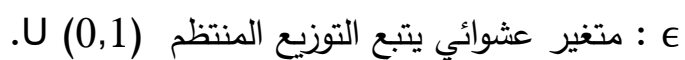

\section{Lévy Flight And Random Walks}

-2-2-2 السير العشوائي و رحلة ليفي

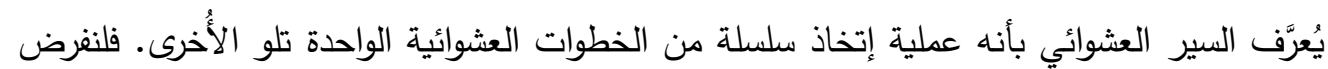

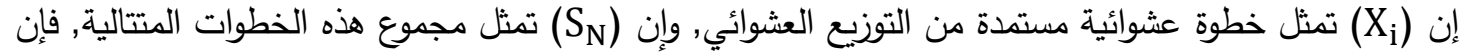
تثكل سيراً عشوائياً كما في المعادلة التالية:

$\mathrm{S}_{\mathrm{N}}=\sum_{\mathrm{i}=1}^{\mathrm{N}} \mathrm{X}_{\mathrm{i}}=\mathrm{X}_{1}+\cdots+\mathrm{X}_{\mathrm{N}}$

ويمكن لحجم الخطوة في السير العشوائي أن يكون ثابتاً أو متفاوتاً][]. أما رحلة ليفي فتُعرف بأنها

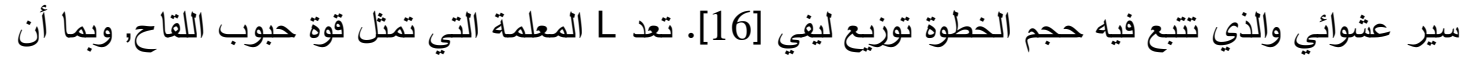

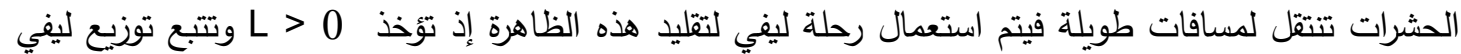
$\mathrm{L} \sim \frac{\lambda \Gamma(\lambda) \sin (\pi \lambda / 2)}{\pi} \frac{1}{\mathrm{~s}^{1+\lambda}}$

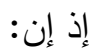

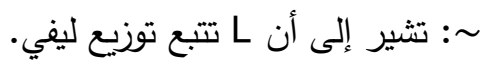

1.5 =

$\mathrm{s}=\frac{\mathrm{U}}{|\mathrm{V}|^{1 / \lambda}}$

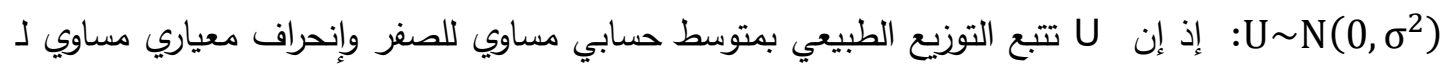
$\cdot \sigma^{2}$

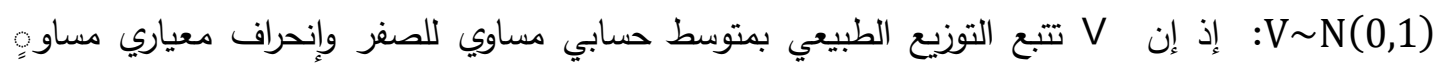
للواحد.
ويتم حساب
$\sigma^{2}=\left[\frac{\Gamma(1+\lambda)}{\lambda \Gamma((1+\lambda) / 2)} \cdot \frac{\sin (\pi \lambda / 2)}{2^{(\lambda-1) / 2}}\right]^{1 / \lambda}$ 
يتم اتباع الخطوات التالية [14]: الخطوة الأولى: توليد المجتمع الأولي عشوائياً لـ (n) من الزهور باستعمال المعادلة الاتية [12]: $\overrightarrow{\mathrm{X}}=\min +\operatorname{rand} *(\max -\min )$

إذ إن: - n

min max rand وتحديد دالة الهدف f(x),

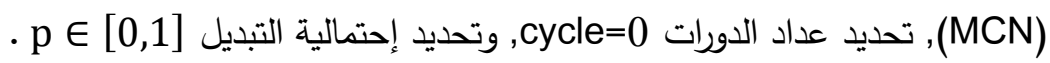
الخطوة الثانية: حساب دالة الهدف لجميع الزهور ويُؤخذ أفضل حل وليكن (XVt) الخطوة الثالثة: فحص معيار التوقف , طالما عداد الدورات cycle أقل من عدد الدورات الكلية (MCN) إنتقل إلى الخطوة الرابعة وإلا توقف.

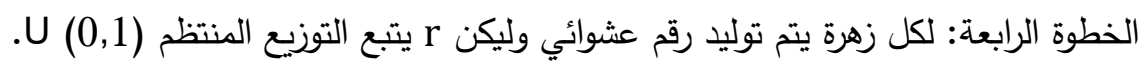
الخطوة الخامسة: إذا كانت r p يتم التلقيح الثامل والذي يتبع توزيع ليفي باستعمال المعادلة (5) وإلا فيتح

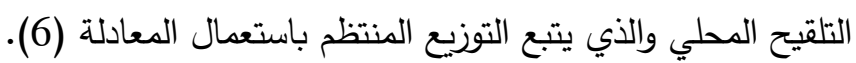

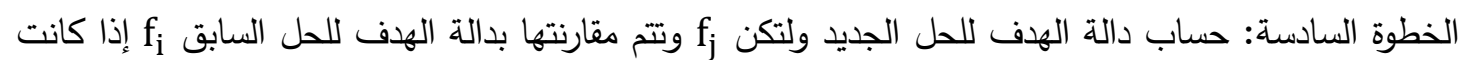

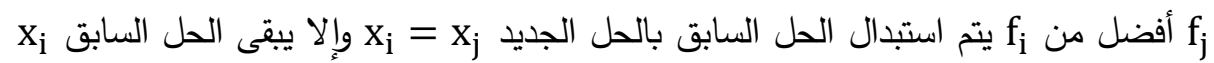
كما هو. الخطوة السابعة: إيجاد أفضل حل جديد وليكن (Xnew-best) ومقارنته مع (X)est) إذا كانت قيمته أفضل فيتم تحديث أفضل حل (X الخطوة الثامنة: تحديث عداد الدورات cycle=cycle+1 والانتقال إلى الخطوة الثالثة. الخطوة التاسعة: Xest يعتبر الحل الأمثل.

\section{Genetic Algorithm(GA)}

تعد الخوارزمية الجينية تقنية بحث دُرِستَ من قبل العالم John Holland سنة 1970. لها القدرة

على حل مسائل الأمثلية المعقدة فخلال العقود الثلاثة المنصرمة إستُعملت الخوارزمية الجينية كخوارزمية تكيفية لحل مسائل عملية, وقد إستُعملت بثكل واسع في العديد من المجالات العلمية [17]. 
GA Cycle

GA 1-2-2

يوضح الثكل(1) المراحل الرئيسية للخوارزمية الجينية [6].

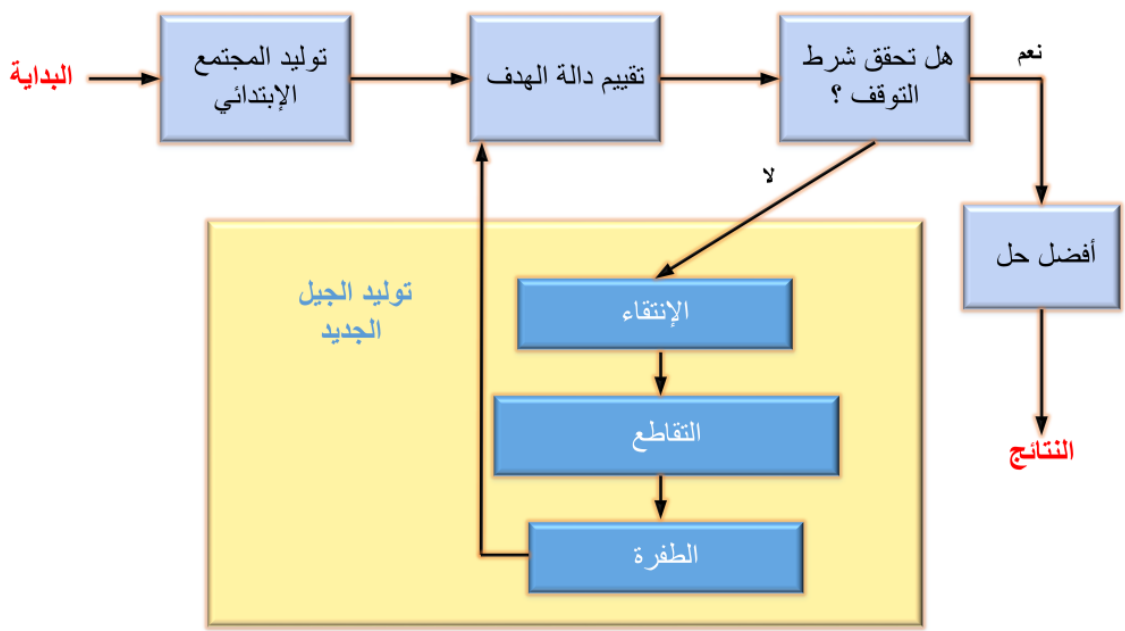

الثكل(1) مراحل الخوارزمية الجينية [18]

للحصول على أفضل الحلول للتطبيق باستعمال الـ (GA) تتبع الخطوات التالية:

Generate Initial Population

1 - توليد المجتمع الإبتدائي

يتم إنثاء المجتمع الأولي X باستعمال المعادلة رقم (11).

Evaluation

التقييم

$-2$

يتم التقييم بتعريف دالة اللياقة لكل كروموسوم. إن دالة اللياقة هذه هي مؤشر للكروموسوم والتي توضح مدى قرب هذا الكروموسوم من الحل المطلوب [13].

Termination Condition

3- شرط التوقف

يفحص شرط التوقف بعد كل جيل لتحديد ما إذا كانت ستستمر الخوارزمية أم تتوقف [18] بالنسبة للبيانات الأولى إذا كان الجيل الحالي G أكبر من Gmax فيتم التوقف, وبالنسبة لمجموعة البيانات الثانية يتم فحص دالة اللياقة كل 10 دورات إذا لم تتغير يتم التوقف.

Generate New Population 4-توليد الجيل الجديد تتمثل عملية توليد الجيل الجديد بالخطوات الثلاث الآتية والمستعملة في هذا البحث [6]:

Selection 1 - إلإختيار أو الإنتقاء

هي عملية إختيار الأفراد من أجل التزاوج وإنتاج جيل جديد ويتم إختيار الأفراد الأكثر صلاحية (أفضل دالة لياقة) لإعادة الإنتاج. 


\section{-: Top- Mate Selection}

يتم إختيار الأب الأول الذي له أفضل دالة لياقة أما الأب الثاني فيتم إختياره عشوائياً.

\section{Crossover}

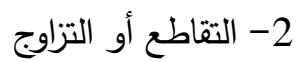

بعد إختيار الآباء بإحدى طرائق الإنتقاء تتم عملية التقاطع وتسمى أيضاً بإعادة الإنتاج. يتم تقاطع

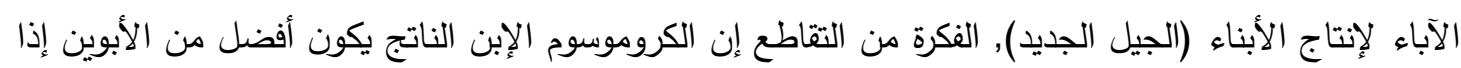
أخذ الصفات الجيدة من كليهما ـ تردد التقاطع يتم التحكم به عن طريق إحتمالية التقاطع ( Crossover)

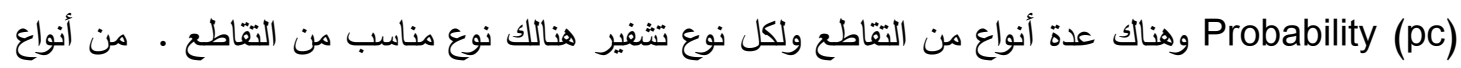

\section{• • ( التقاطع الحدسي (Heuristic Crossover):}

يستعمل مع التشفير الحقيقي إذ يعتمد على قيمة اللياقة للأبوين لإنتاج الأبناء, يتم توليد إبن

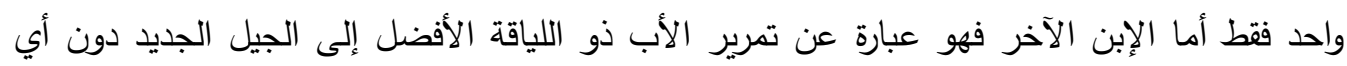
معالجة, والمعادلات الآتية توضح هذا الآنوع من التقاطع :

fitness $_{\text {parent1 }}$ is better than fitness parent2

$$
\text { offspring }_{1}=\text { parent }_{1}
$$

offspring $_{2}=$ parent $_{1} \pm \mathrm{r} *\left(\right.$ parent $_{1}-$ parent $\left._{2}\right)$

$$
\text { U رقم عشوائي يتبع التوزيع المنتظم r r }
$$

$$
\text { 3- الطفرة }
$$

Mutation

تتم عملية الطفرة بعد مرحلة التقاطع, وهي عبارة عن تغيير في قيمة الجين الواحد أو أكثر للكروموسوم بالإعتماد على إحتمالية الطفرة (Mutation Probability (pm) والتي تكون صغيرة عادةً. عملية الطفرة تحافظ على التتوع في الجيل إذ تؤدي إلى إنتاج حلول جديدة متتوعة وهذا يزيد من سرعة الإقتراب إلى

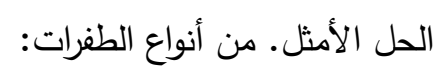

الطفرة غير المنتظمة (Non-Uniform Mutation): يستعمل في التشفير الحقيقي إذ قيمة الكروموسوم الأب تتغير في نطاق محدود بالنظر إلى رقم الجيل الحالي, فإذا كان رقم الجيل الحالي

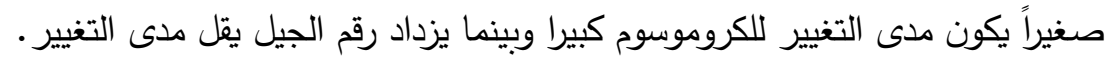
offspring $_{\mathrm{i}}=\left\{\begin{array}{c}\text { parent }_{\mathrm{i}}+\left(\text { upperbound }_{\mathrm{i}}-\text { parent }_{\mathrm{i}}\right) * \mathrm{f}(\mathrm{G}) \\ \text { or } \\ \text { parent }_{\mathrm{i}}-\left(\text { parent }_{\mathrm{i}}-\text { lowerbound }_{\mathrm{i}}\right) * \mathrm{f}(\mathrm{G})\end{array}\right\} \ldots$ upperbound lowerbound f(G) : دالة مدى التغيير والتي تحسب من المعادلة: 
$f(G)=\left(r *\left(1-\frac{G}{G_{\max }}\right)\right)^{b}$

إذ إن

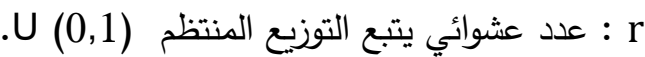

Gmax

G

معلمة الحالة والتي تحدد درجة الإعتمادية على الجيل الحالي. b

\section{Algorithm Steps}

2-2-2

يتم اتباع الخطوات التالية [19]: ع

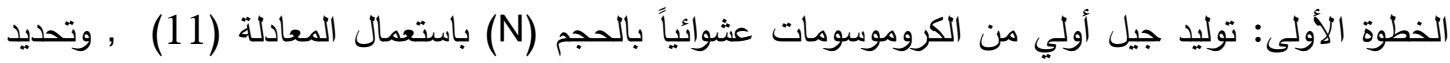
دالة الهدف f(x), x = (x

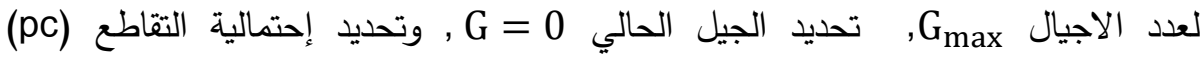

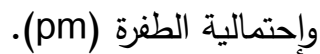

الخطوة الثانية: حساب دالة الهدف لجميع الكروموسومات.

الخطوة الثالثة: فحص معيار التوقف, طالما لم يتحقق شرط التوقف إنتقل إلى الخطوة الرابعة وإلا توقف. الخطوة الرابعة: يتم إختيار أبوين لإنتاج الأبناء باستعمال طريقة (Top-Mate Selection). الخطوة الخامسة: توليد عدد عشوائي pcross , إذا كانت pcross > pc يتم التقاطع باستعمال طريقة التقاطع الحسسي.

الخطوة السادسة: توليد عدد عشوائي pmut , إذا كانت pmut > pm تتم الطفرة باستعمال الطفرة غير

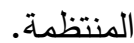

الخطوة السابعة : وضع الكروموسومات الناتجة (الأبناء) في الجيل الجديد.

الخطوة الثامنة: طالما حجم الجيل الجديد أقل من N, إنتقل إلى الخطوة الرابعة وإلا إنتقل إلى الخطوة التاسعة. الخطوة التاسعة: إبدال الجيل السابق بالجيل الحالي وتحديث الجيل الحالي 1 G = G و الإنتقال إلى الخطوة الثانية.

\section{Proposed Hybrid Algorithm}

2-2 الخوارزمية المهجنة المقترحة

لغرض تحسين اداء خوارزمية تلقيح الزهرة تم تهجينها في هذا العمل مع الخوارزمية الجينية المشفرة

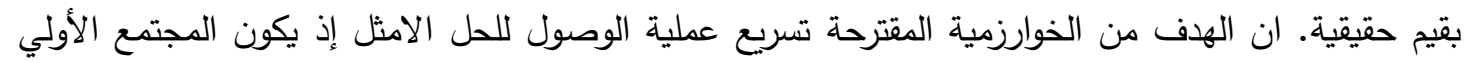

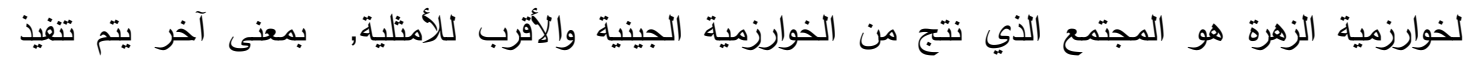
الخوارزمية الجينية أولاً والمجتمع الذي يتم الحصول عليه عند تحقق شرط التوقف يُعيّن كمجتمع إبتدائي لخوارزمية الزهرة ثم تنفذ الخوارزمية للحصول على أفضل حله 


\section{النتائج والمناقشة}

النتائج عند استعمال البيانات الأُولى:

FPA 1 - Fتائج تنفيذ خوارزمية

تمت مقارنة نتائج تتفيذ خوارزمية الـ (FPA) للبيانات الأولى و بنسبة 70\% للتدربب و 30\%

للإختبار مع النتائج المذكورة في كل من [11] و [12]. الجدول (3) يوضح نتائج تتفيذ الخوارزمية مع أفضل

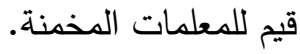

الجدول (3): مقارنة نتائج FPA مع نتائج PSO, ABC, DABC للبيانات الأولى

\begin{tabular}{|c|c|c|c|c|c|c|}
\hline \multirow{2}{*}{ Model } & \multicolumn{4}{|c|}{ RMSE-testing (30\%of data) } & \multicolumn{2}{c|}{ Best values by FPA } \\
\cline { 2 - 7 } & PSO & ABC & DABC & FPA & A & b \\
\hline G_O & 80.8963 & 119.6425 & $\mathbf{7 2 . 0 1 8 7}$ & 77.8622 & 684.3160 & 0.0174 \\
\hline POW & 149.9684 & 158.6753 & 81.9231 & $\mathbf{1 4 6 . 3 8 1 2}$ & 22.3832 & 0.7281 \\
\hline DSS & 17.0638 & 17.091 & 29.8051 & $\mathbf{1 6 . 6 2 7 7}$ & 501.8481 & 0.0636 \\
\hline
\end{tabular}

إذ تفوقت خوارزمية (PPA) على خوارزمية (PSO) و (ABC) لجميع النماذج وتفوقت على (DSO)

في نموذج الـ (DSS) فقط. وقد تم تحديد أفضل القيم بالخط الغامق.

\section{2- نتائج تنفيذ خوارزمية تلقيح الزهرة المهنة HFPA:}

تم تنفيذ الخوارزمية المهجنة للبيانات الأولى ولجميع النماذج ومقارنة النتائج مع خوارزمية (FPA) الإعتيادية وكما هو ملاحظ من الجدول (4) إن (HFPA) توصلت تقريباً إلى قيمة (RMSE) نفسها بعدد (RPA)

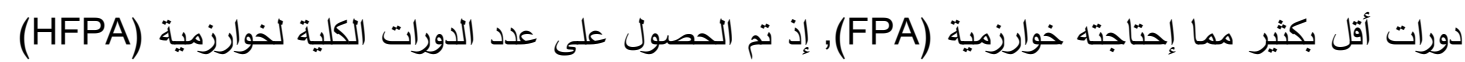
من خلال جمع عدد دورات الـ (RGA) مع عدد دورات الـ (FPA). 
الجدول (4): مقارنة نتائج HFPA مع نتائج FPA للبيانات الأولى

\begin{tabular}{|c|c|c|c|c|c|c|c|}
\hline \multirow[b]{2}{*}{ Model } & \multicolumn{2}{|c|}{ FPA } & \multicolumn{4}{|c|}{ Hybrid FPA } & \multirow{2}{*}{$\begin{array}{c}\text { Improve- } \\
\text { ment }\end{array}$} \\
\hline & $\begin{array}{l}\text { RMSE- } \\
\text { testing }\end{array}$ & $\begin{array}{l}\text { No. Of } \\
\text { cycles }\end{array}$ & $\begin{array}{l}\text { RMSE- } \\
\text { testing }\end{array}$ & $\begin{array}{c}\text { No. Of } \\
\text { cycles by } \\
\text { RGA }\end{array}$ & $\begin{array}{l}\text { No. Of } \\
\text { cycles } \\
\text { by FPA }\end{array}$ & $\begin{array}{l}\text { Total no. } \\
\text { of cycles } \\
\text { by HFPA }\end{array}$ & \\
\hline G_O & 77.862 & 508 & 77.859 & 50 & 316 & 366 & $27.95 \%$ \\
\hline POW & $\begin{array}{c}146.38 \\
1\end{array}$ & 591 & $\begin{array}{c}146.78 \\
5\end{array}$ & 80 & 491 & 571 & $3.38 \%$ \\
\hline DSS & 16.627 & 480 & 16.627 & 40 & 197 & 237 & $50.62 \%$ \\
\hline
\end{tabular}

النتائج عند استعمال مجموعة البيانات الثانية:

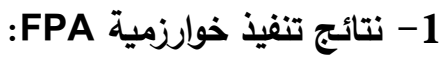

لكل نموذج من البيانات الثلاثة تم تنفيذ كل خوارزمية 100 مرة ثم إختيار أقل (MSE) تم الحصول

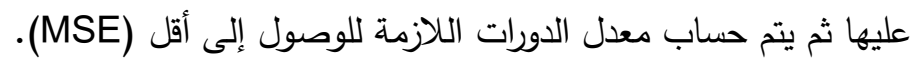

الجدول (5) مقارنة نتائج FPA مع نتائج CGA,MGA لمجموعة البيانات الثانية

\begin{tabular}{|c|c|c|c|c|c|c|c|c|c|c|}
\hline \multirow{2}{*}{$\begin{array}{c}\text { Data- } \\
\text { set }\end{array}$} & \multirow[b]{2}{*}{ Model } & \multicolumn{2}{|c|}{ CGA } & \multicolumn{2}{|c|}{ MGA } & \multicolumn{2}{|c|}{ FPA } & \multicolumn{3}{|c|}{ Best values found by FPA } \\
\hline & & MSE & $\begin{array}{l}\text { Av. No. } \\
\text { Of gen. }\end{array}$ & MSE & $\begin{array}{l}\text { Av. No. } \\
\text { Of gen. }\end{array}$ & MSE & $\begin{array}{c}\text { Av. No. } \\
\text { Of } \\
\text { gen. }\end{array}$ & A & $b$ & C \\
\hline \multirow{2}{*}{ DS1 } & G_O & 139.815 & 13415 & 139.815 & 5341 & 139.815 & 759 & 760.534 & 0.032 & - \\
\hline & INFS & 82.704 & 78664 & 82.704 & 15664 & 82.704 & 809 & 382.373 & 0.178 & 2.886 \\
\hline \multirow{2}{*}{ DS2 } & G_O & 11.617 & 48104 & 11.617 & 7331 & 11.617 & 299 & 130.201 & 0.083 & - \\
\hline & INFS & 8.98 & 77889 & 8.98 & 17490 & 8.980 & 616 & 110.631 & 0.173 & 1.221 \\
\hline \multirow{2}{*}{ DS3 } & G_O & 22.863 & 39209 & 22.863 & 3350 & 20.391 & 325 & 1000 & 0.006 & - \\
\hline & INFS & 5.82 & 83578 & 5.82 & 20749 & 5.820 & 889 & 229.198 & 0.087 & 3.703 \\
\hline
\end{tabular}


2-2 نتائج تنفيذ خوارزمية تلقيح الزهرة المهجنة HFPA:

تم تنفيذ الخوارزمية المهجنة لمجموعة البيانات الثانية ولجميع النماذج إذ تم التتفيذ لـ 100 مرة وفي كل تتفيذ تم الحصول على قيمة (MSE) وعلى عدد الدورات اللازمة للوصول الى هذه القيمة (عدد الدورات لـ RGA الخوارزمية المهجنة للوصول الى قيمة (MSE). بعد انتهاء جميع التنفيذات يتم إختيار أقل (MSE) تم الحصول

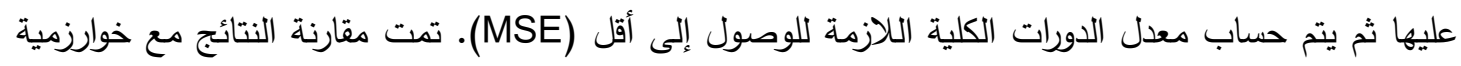

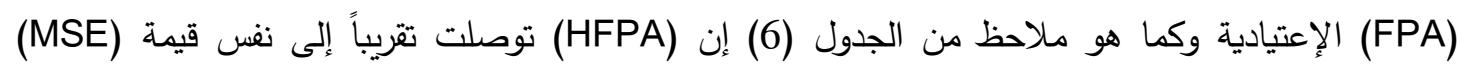
بمعدل دورات أقل بكثير مما إحتاجته خوارزمية (FPA).

الجدول (6) مقارنة نتائج HFPA مع نتائج FPA لمجموعة البيانات الثانية

\begin{tabular}{|c|c|c|c|c|c|c|c|c|}
\hline $\begin{array}{c}\text { Data } \\
\text { set }\end{array}$ & Model & \multicolumn{2}{|c|}{ FPA } & \multicolumn{4}{c|}{ HFPA } & $\begin{array}{c}\text { Improve- } \\
\text { ment }\end{array}$ \\
\hline \multirow{2}{*}{ DS1 } & & \begin{tabular}{c} 
Av. \\
MSE- \\
testing \\
Of \\
\cline { 2 - 9 }
\end{tabular} & Gen. & $\begin{array}{c}\text { MSE- } \\
\text { testing }\end{array}$ & $\begin{array}{c}\text { No. Of } \\
\text { cycles } \\
\text { by RGA }\end{array}$ & $\begin{array}{c}\text { No. Of } \\
\text { cycles } \\
\text { by FPA }\end{array}$ & $\begin{array}{c}\text { No. Of } \\
\text { gen. } \\
\text { by } \\
\text { HFPA }\end{array}$ & \\
\hline \multirow{2}{*}{ DS2 } & 139.815 & 759 & 139.815 & 60 & 382 & 527 & $30.56 \%$ \\
\cline { 2 - 10 } & INFS & 82.704 & 809 & 82.704 & 40 & 240 & 419 & $48.20 \%$ \\
\hline \multirow{2}{*}{ DS3 } & 11.617 & 299 & 11.617 & 20 & 46 & 86 & $71.23 \%$ \\
\cline { 2 - 9 } & G-O & 20.391 & 325 & 20.429 & 20 & 6 & 23 & $92.92 \%$ \\
\hline
\end{tabular}


وفيما يأتي المقطع البرمجي الخاص بخوارزمية تلقيح الزهرة FPA:

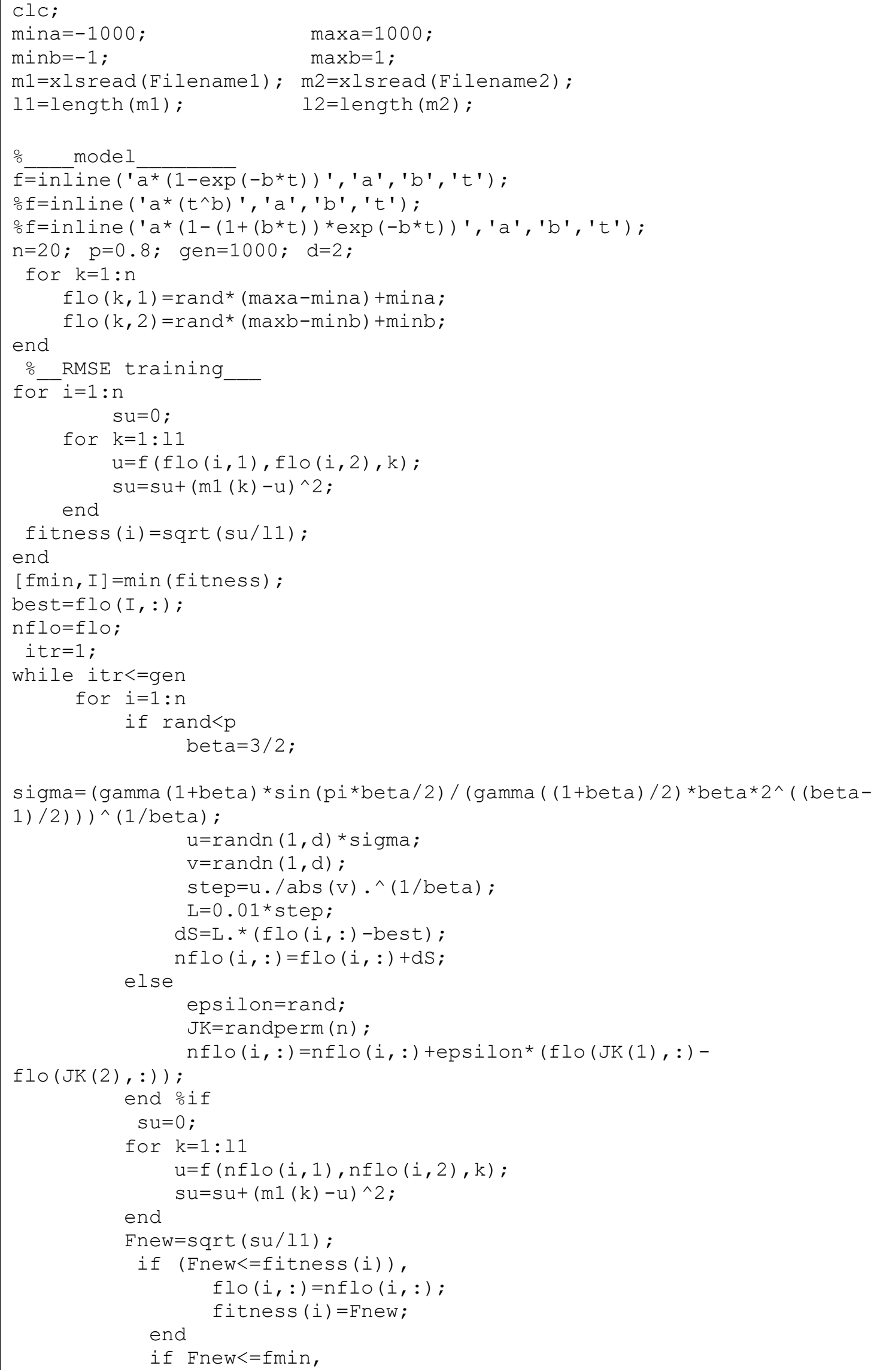




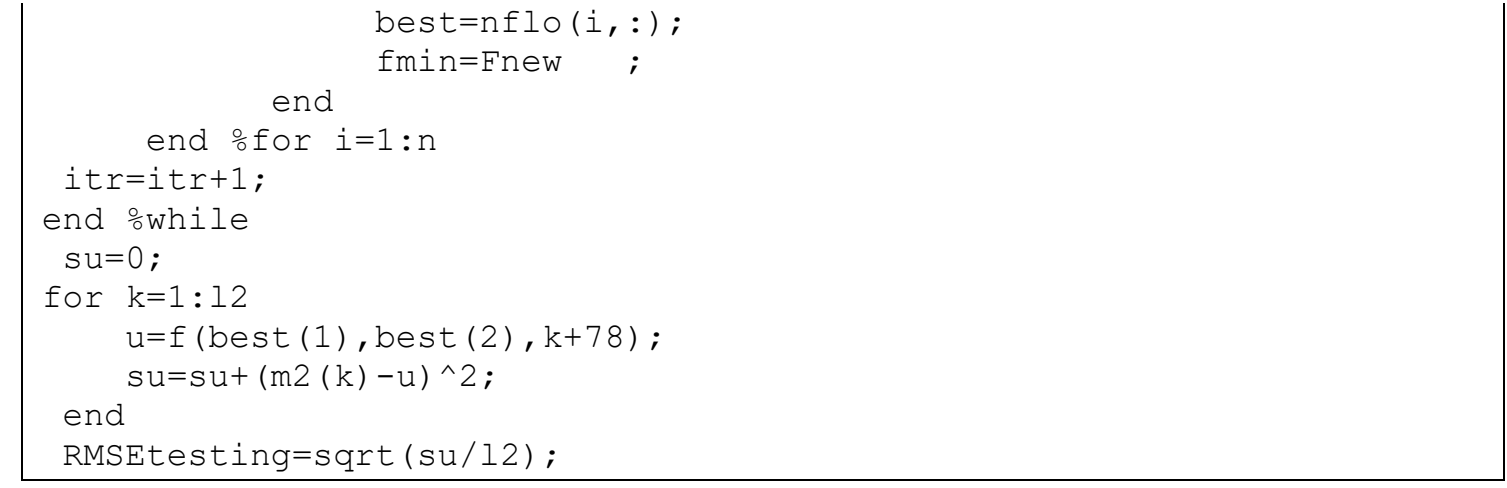

\section{المقطع البرمجي الخاص بخوارزمية الزهرة المهنة HFPA كالاتي:}

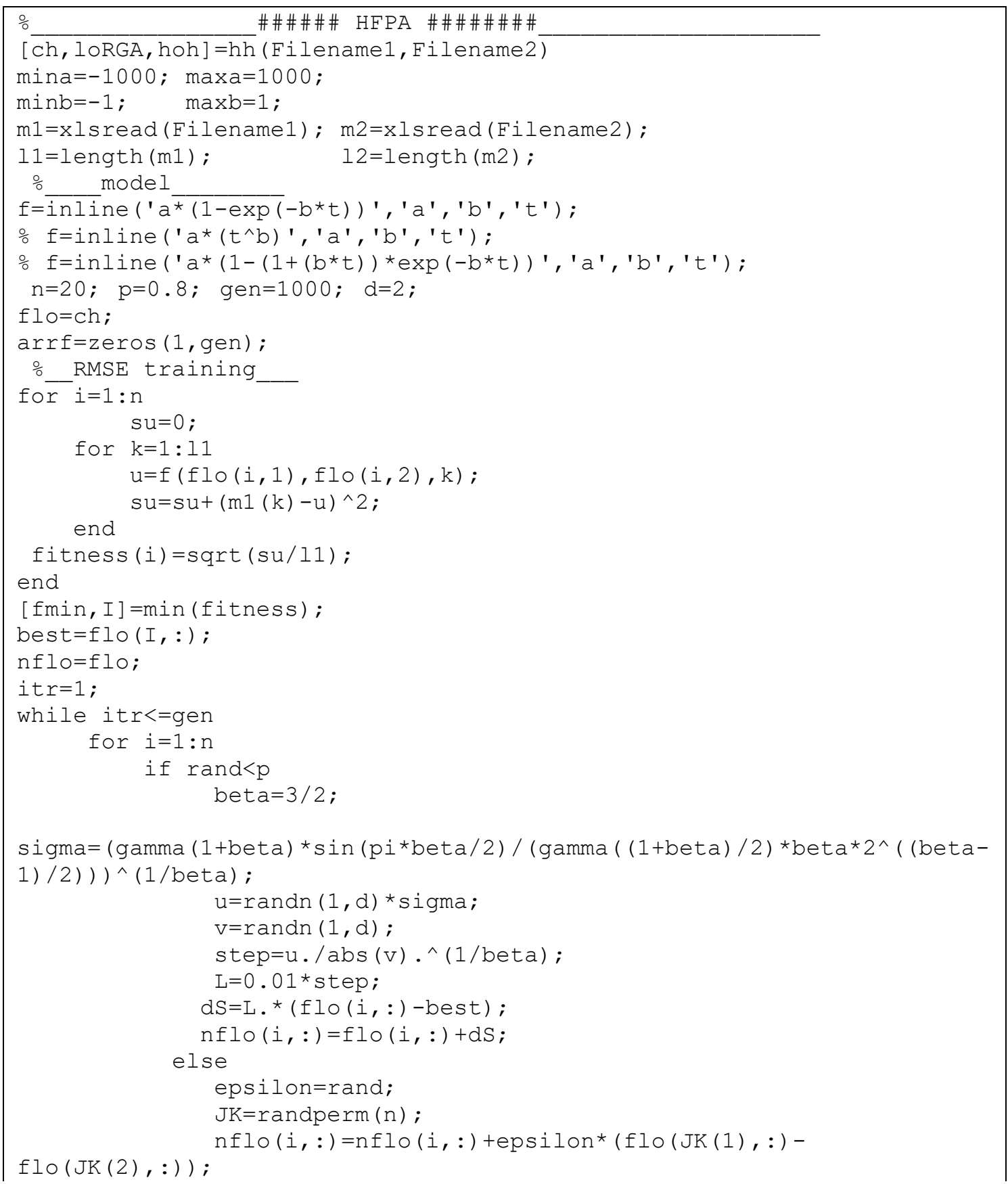




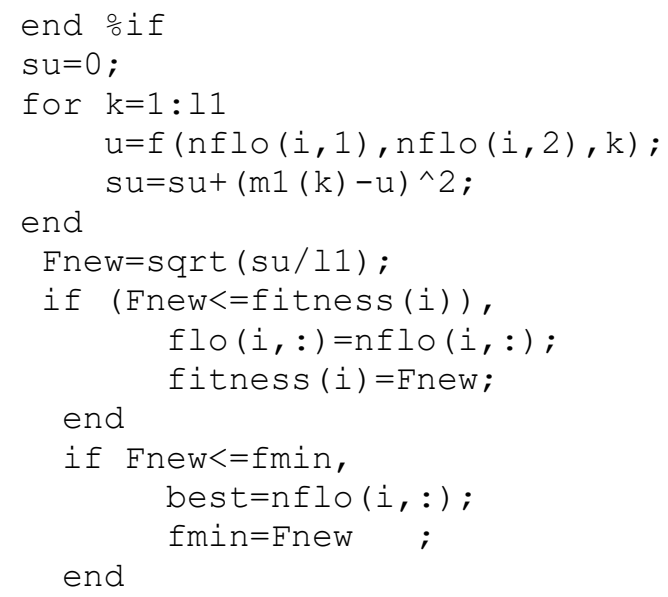

end $\frac{\circ}{0}$ or $i=1: n$

itreitr+1;

end $\%$ while

$\mathrm{su}=0$;

for $k=1: 12$

$\mathrm{u}=\mathrm{f}($ best $(1)$, best (2), $\mathrm{k}+78)$;

$\mathrm{su}=\mathrm{su}+(\mathrm{m} 2(\mathrm{k})-\mathrm{u})^{\wedge} 2$;

end

RMSEtesting=sqrt (su/12);

$\frac{\circ}{0}$

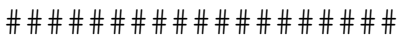
GA

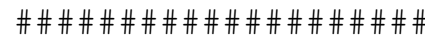

function [ch, loRGA, hoh ] hh (Filename1, Filename2)

mina $=-1000 ; \operatorname{maxa}=1000$;

$\operatorname{minb}=-1 ; \quad \operatorname{maxb}=1$;

$\mathrm{m} 1=\mathrm{xl}$ sread (Filename1); $\mathrm{m} 2=x \mathrm{lsread}(\mathrm{Filename2)}$;

$11=$ length $(\mathrm{m} 1) ; \quad 12=$ length $(\mathrm{m} 2)$;

o model

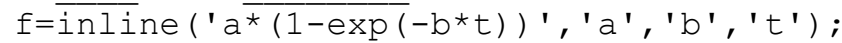

\% $f=i n l i n e\left(a^{*}\left(t^{\wedge} b\right)^{\prime},{ }^{*} a^{\prime}, b^{\prime} b^{\prime}, t^{\prime}\right)$;

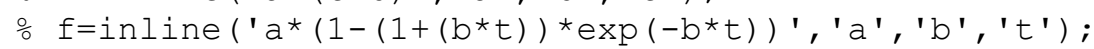

initial

size=20; gen=1000; po=0;

ch=zeros (size, 2);

for $k=1:$ size

ch $(k, 1)=r a n d *($ maxa-mina $)+$ mina;

end

ch $(k, 2)=\operatorname{rand}^{*}(\operatorname{maxb}-\mathrm{minb})+$ minb;

$\mathrm{I}=1$;

ogeneration

while $(I<=g e n)$

num $=$ size;

$t=0$;

for $j=1$ :size end fitt $(j)=f i t t n e s s(c h(j, 1), \operatorname{ch}(j, 2), 11, m 1, f)$;

oselection

while (num>=2)

[val seq] =min (fitt);

$\mathrm{p} 1=\mathrm{ch}($ seq, : ) ;

h=randperm (num);

for $e=1$ : num

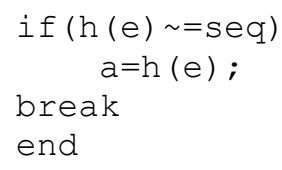


off $1=\mathrm{p} 1$;

end

$\mathrm{p} 2=\mathrm{ch}(\mathrm{a},:) ;$

off $2=\mathrm{p} 2$;

if $($ rand $<0.5)$

ocross

[off1, off2] = crossover $(\mathrm{p} 1, \mathrm{p} 2)$;

end

omutation

pmut $=$ rand;

if (pmut<0.1)

off=mutation (off2, I, gen, maxa, mina, maxb,minb) ; end

ostore

$t=t+1$

$\operatorname{newch}(t,:)=$ offl;

newfitt $(t)=$ fittness $(\operatorname{offl}(1,1), \operatorname{offl}(1,2), 11, \mathrm{~m} 1, \mathrm{f})$;

$t=t+1$

$\operatorname{newch}(t,:)=\operatorname{off} 2$;

newfitt $(t)=$ fittness $(\operatorname{off} 2(1,1), \operatorname{off} 2(1,2), 11, \mathrm{ml}, \mathrm{f})$;

odelete from ch and fitt

dch=inf (size, 2);

dfitt=inf (size, 1$)$;

$\mathrm{z}=0$;

for $\mathrm{fd}=1$ : num

if $((\mathrm{fd} \sim=\mathrm{a}) \& \&(\mathrm{fd} \sim=\mathrm{seq}))$

$\mathrm{z}=\mathrm{z}+1$;

$\operatorname{dch}(z,:)=\operatorname{ch}(f d,:)$;

$\operatorname{dfitt}(z)=$ fitt $(\mathrm{fd})$;

end

end

ch=zeros (size, 2$)$;

fitt $=\operatorname{zeros}(\operatorname{size}, 1)$;

ch=dch;

fitt=dfitt;

num $=$ num -2 ;

end $\frac{\circ}{\circ}$ while (num>=2)

ch=newch;

fitt=newfitt;

newch=zeros (size, 2);

newfitt=zeros (size, 1$)$;

if $\operatorname{round}(I / 10)==I / 10$,

[va ya] $=\min$ (fitt) ;

$\mathrm{po}=\mathrm{po}+1$;

$\mathrm{gg}(\mathrm{po})=\mathrm{va}$;

if $p o>1$

$\mathrm{z} 1=\mathrm{abs}(\mathrm{va}-\mathrm{gg}(\mathrm{po}-1))$;

if $(\mathrm{z} 1<0.0001)$

break

end

end

end

$\mathrm{I}=\mathrm{I}+1$;

end ond while ( $<<=$ gen)

$[\operatorname{val} y]=\min$ (fitt) ;

응

RMSE testing

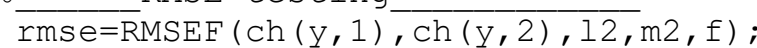

응

crossover

function $[$ offl, off $\overline{2}]=\operatorname{crossover}(\mathrm{p} 1, \mathrm{p} 2)$ 


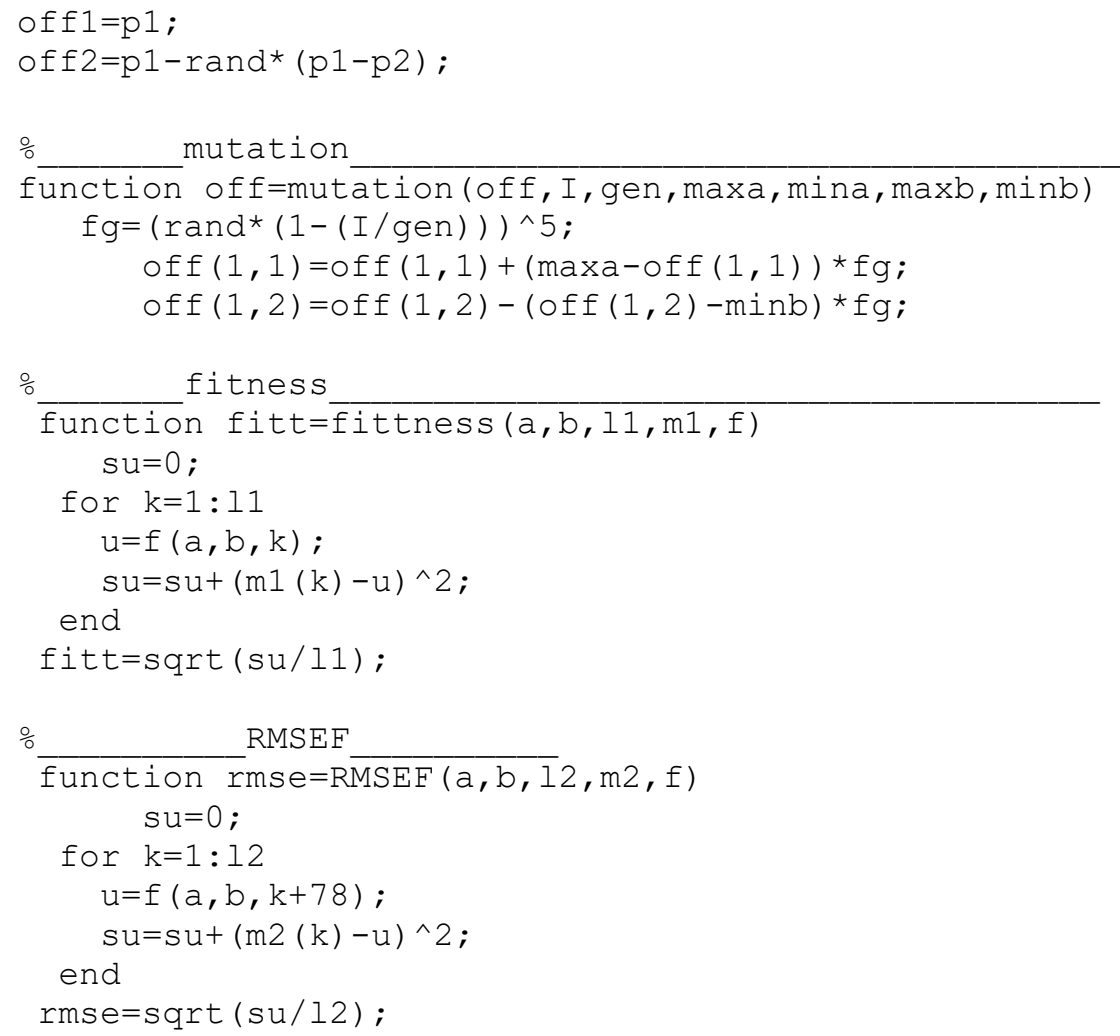

\section{الاستتتاجات والتوصيات}

هنالك عدة طرائق تقليدية لتخمين معلمات نماذج الـ (SRGMs) لكنها تعاني من عدة مشاكل, لذلك

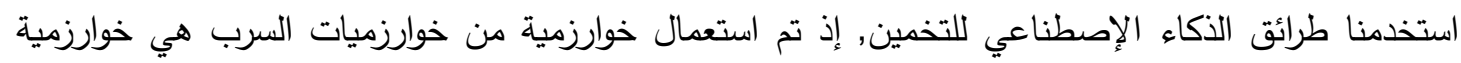
تلقيح الزهرة, وكذلك تم استعمال خوارزمية تطورية وهي الخوارزمية الجينية الحقيقية, ثم تم تهجين الخوارزمية

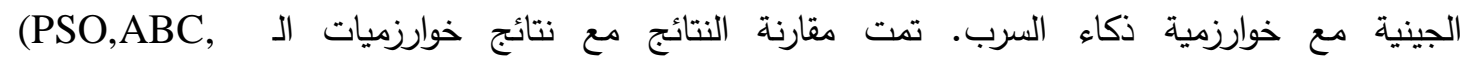

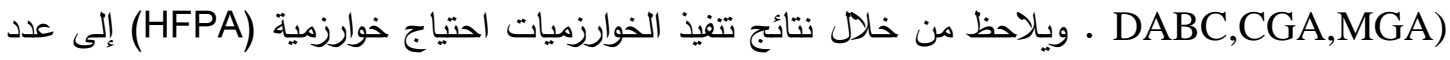
دورات أقل مما إحتاجته خوارزمية (FPA) للوصول إلى أفضل حل, إذ تناقصت عدد الدورات في الخوارزمية المهجنة بالنسب المذكورة في الجدولين (4) و (6). 


\section{(لمصادر}

[1] Sheakh, T. H. and Singh, V. P., "Taxonomical Study Of Software Reliability Growth Models", International Journal of Scientific and Research Publications, Volume 2, Issue 5, pp.1-3 (2012).

[2] Wood, A., "Software Reliability Growth Models", Tandem Tech., Technical Report, Vol. 96.1, Tandem Computers Inc., Corporate Information Center, Cupertino Calif., Part Number 130056 (1996).

[3] Al-Saati, N. A. and Abd-Alkareem, M., " The Use of Cuckoo Search in Estimating the Parameters of Software Reliability Growth Models", (IJCSIS) International Journal of Computer Science and Information Security, Vol. 11, No. 6, (2013).

[4] Mirjalili, S. A. and Mirjalili , S. M., Lewis, A., "Grey Wolf Optimizer", Advances in Engineering Software 69, pp. 46-61(2014).

[5] Kelanibandara, K.W.K.B.P.L.M., "Software Reliability Estimation Using Cubic Splines Network Model", M.Sc. thesis, School of Computing, University of Colombo, (2012).

[6] Alneamy, J. S. and Dabdoob, M. M., "The Use of Original and Hybrid Grey Wolf Optimizer in Estimating the Parameters of Software Reliability Growth Models", International Journal of Computer Applications, Volume 167-No.3, pp.1221(2017).

[7] Lai, R. and Garg, M., "A Detailed Study of NHPP Software Reliability Models", Journal of Software, Vol.7, No.6, pp.1296-1306 (2012).

[8] Meyfroyt, P. H. A., "Parameter Estimation for Software Reliability Models", M.Sc. thesis, Eindhoven: Technische Universiteit Eindhoven, (2012).

[9] Shanmugam, L. and Florence, L., "A Comparison of Parameter Best Estimation Method for Software Reliability Models", International Journal of Software Engineering \& Applications (IJSEA), Vol.3, No.5, pp.91-102 (2012).

[10] Prasad, R. S., Rao, K. P. and Mohan, G. K., "Software Reliability using SPRT: Inflection S-shaped Model", International Journal of Application or Innovation in Engineering \& Management, Volume 2, Issue 6, pp. 349-355 (2013).

[11] Sheta, A. F., " Parameter Estimation of Software Reliability Growth Models by Particle Swarm Optimization", AIML Journal, Volume (7), Issue (1), pp.5561(2007).

[12] Sharma, T.K., Pant, M. and Abraham, A., "Dichotomous Search in ABC and its Application in Parameter Estimation of Software Reliability Growth Models ", Third World Congress on Nature and Biologically Inspired Computing, pp.214219 (2011).

[13] Hsu, C.J. and Huang, C.Y., "A Study on the Applicability of Modified Genetic Algorithms for the Parameter Estimation of Software Reliability Modeling " , IEEE 34th Annual Computer Software and Applications Conference, pp.531540 (2010). 
[14] Yang, X. S., "Flower Pollination Algorithm for Global Optimization", in: Unconventional Computation and Natural Computation, Lecture Notes in Computer Science, Vol. 7445, pp. 240-249 (2013).

[15] Yang, X. S., Karamanoglu, M. and He, X. S., "Flower Pollination Algorithm: A Novel Approach for Multiobjective Optimization", Engineering Optimization, vol. 46, Issue 9, pp. 1222-1237 (2014).

[16] Yang, X. S., "Nature Inspired Metahuristic Algorithms", Second Edition, Luniver Press, pp:1-160 (2010).

[17] HERRERA, F., LOZANO, M. and VERDEGAY, J.L., "Tackling Real Coded Genetic Algorithms: Operators and Tools for Behavioural Analysis", Artificial Intelligence Review 12: 265-319 (1998).

[18] AL Neamy, J. S., "Brain Tumors Images Diagnosis Using Hybrid Intelligency Techniques", Ph.D. Thesis, college of computers and mathematics science, University of Mosul (2006).

[19] MENDES, J. M., "A Comparative Study of Crossover Operators for Genetic Algorithms to Solve the Job Shop Scheduling Problem", WSEAS TRANSACTIONS on COMPUTERS, Issue 4, Volume 12, pp. 164-173 (2013). 